\title{
Myélome Multiple Du Sujet Jeune Révélé Par Un Plasmocytome Sacré: À Propos D'un Cas
}

\author{
Moyikoua Régis Franck, \\ Service d'imagerie médicale, CHU de Brazzaville, Congo \\ Lamini N'Soundhat Norbert Edgard, \\ Service de rhumatologie, CHU de Brazzaville, Congo \\ Ngolet Ocini Lydie, \\ Service d'hématologie, CHU de Brazzaville, Congo
}

Kinata Bambino Sinclair,

Service de chirurgie polyvalente, CHU de Brazzaville, Congo

Doi:10.19044/esj.2019.v15n33p374 URL:http://dx.doi.org/10.19044/esj.2019.v15n33p374

\section{Résumé}

Objectif : Les auteurs rapportent un cas de myélome multiple survenu chez un adulte jeune, révélé par un plasmocytome du sacrum. L'intérêt de cette observation est diagnostic car les tumeurs du sacrum chez le sujet jeune sont rares et posent un problème de diagnostic différentiel avec le chordome et les métastases osseuses. Par ailleurs, la détection des lésions osseuses myélomateuses, étape indispensable au moment du diagnostic, se fait plus facilement au scanner qu'avec la radiographie conventionnelle.

Mots clés : Myélome Multiple, Adulte Jeune, Plasmocytome, Sacrum 


\title{
Multiple Myeloma in a Young Adult Revealed by Sacral Plasmacytoma: About a Case
}

\author{
Moyikoua Regis Franck, \\ Service d'imagerie médicale, CHU de Brazzaville, Congo \\ Lamini N'Soundhat Norbert Edgard, \\ Service de rhumatologie, CHU de Brazzaville, Congo \\ Ngolet Ocini Lydie, \\ Service d'hématologie, CHU de Brazzaville, Congo
}

Kinata Bambino Sinclair,

Service de chirurgie polyvalente, CHU de Brazzaville, Congo

\begin{abstract}
Objective: The authors report a case of multiple myeloma in the young adult revealed by a sacrum's plasmacytoma. The purpose of this observation is diagnostic because sacral tumors in young adult are rare and create an issue of differential diagnosis with the Chordoma and bone metastases. In addition, detecting myeloma bone lesions, an essential step at the time of diagnosis, is easier with CT-scan than traditional radiographies.
\end{abstract}

Keywords: Multiple Myeloma, Young Adult, Plasmacytoma, Sacrum

\section{Introduction}

Le myélome multiple des os est un cancer d'origine hématologique qui survient rarement chez le sujet jeune. Il se caractérise par la prolifération de plasmocytes anormaux au sein de la moelle osseuse et par la formation de multiples lésions ostéolytiques appelés plasmocytomes. Cet article rapporte un cas de myélome multiple survenu chez un adulte jeune, révélé par un volumineux plasmocytome sacré associé à de multiples lésions lytiques du squelette axial.

\section{Observation}

Il s'agit de l'observation d'un patient de 32 ans, admis dans le service de rhumatologie du CHU de Brazzaville pour la prise en charge de douleurs lombo-sacrées invalidantes évoluant depuis deux mois. La douleur était lancinante, fixe, d'intensité rapidement croissante cotée à 6 à l'échelle visuelle analogique, empêchant la station assise prolongée sans fièvre ni troubles sphinctériens associés. 
Le patient ne présentait aucun antécédent médico-chirurgical particulier.

A l'examen clinique, son état général était conservé, il existait un syndrome rachidien lombo-sacré sans déficit moteur ou sensitif des membres pelviens.

Le bilan biologique révélait un syndrome inflammatoire biologique avec une vitesse de sédimentation à $88 \mathrm{~mm}$ à la première heure et une CRP à $24 \mathrm{mg} / \mathrm{L}$. La créatininémie et la calcémie étaient élevés, dosées respectivement à $280 \mu \mathrm{mol} / \mathrm{L}$ et $4,4 \mathrm{mmol} / \mathrm{L}$. L'hémogramme était normal.

Les radiographies du rachis lombo-sacré montraient la présence d'un processus lésionnel ostéolytique centimétrique à contours flous, centré sur le sacrum (fig. 1).

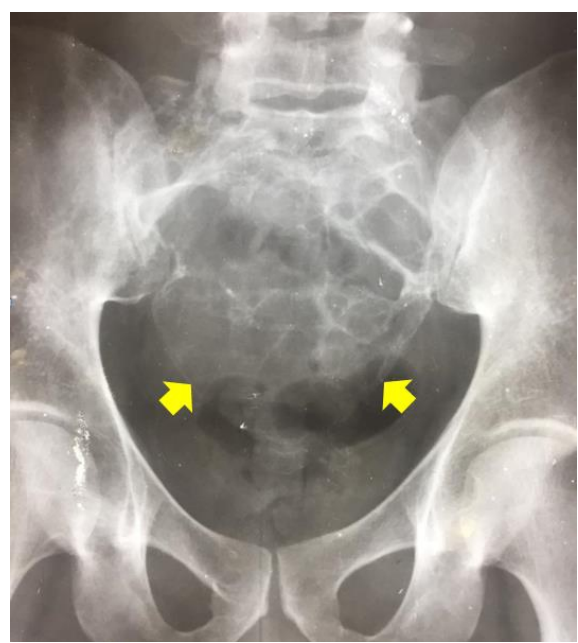

Figure 1 : Radiographie de face centrée sur le sacrum montrant une ostéolyse massive des pièces sacrées (flèches jaunes)

Le scanner thoraco abdomino pelvien mettait en évidence une lésion tumorale du sacrum, de densité tissulaire, hétérogène, bien circonscrite, mesurant $98 \mathrm{~mm}$ de grand axe (fig. $2 \mathrm{a}$ et $2 \mathrm{~b}$ ). 


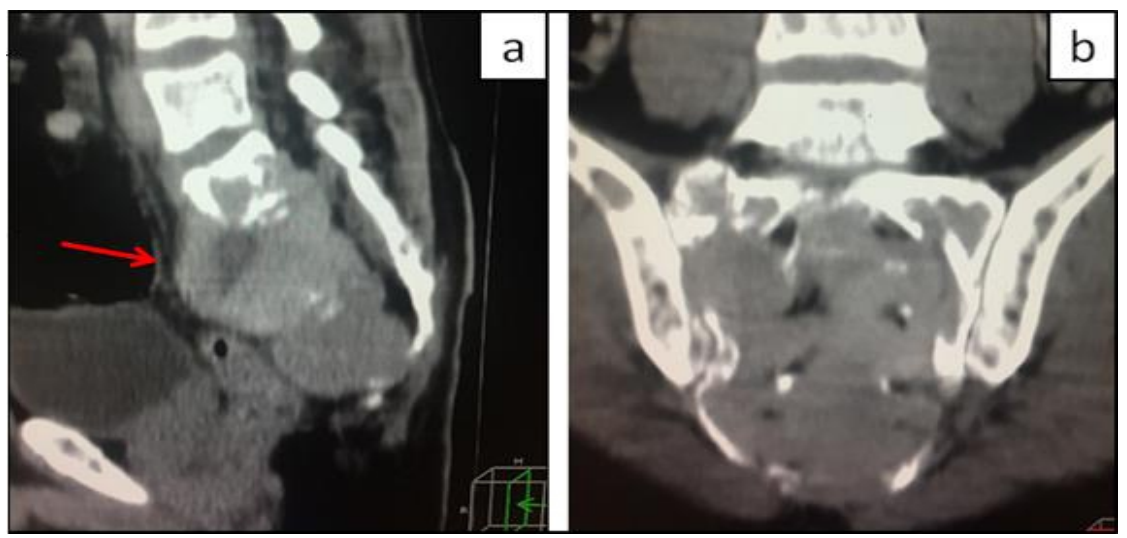

Figures 2 a et b : coupes scannographiques sagittale (a) et coronale (b) en filtre mou du sacrum montrant la lésion tissulaire détruisant les vertèbres sacrées (flèche rouge)

La tumeur détruisait la quasi-totalité des vertèbres sacrées et s'étendait partiellement en avant à la fosse sacrée et en arrière vers le canal rachidien sans compression évidente du filum terminal. Il s’y associait la présence de multiples lésions ostéolytiques, centimétriques à l'emporte-pièce, diffuses au squelette axial, non objectivées par les radiographies standards (fig. 3a et 3b).

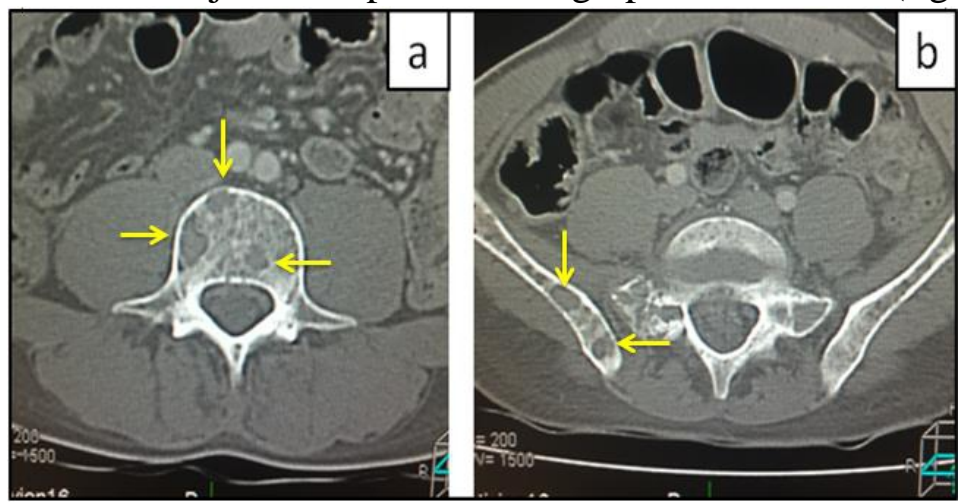

Figure 3 a et $\mathbf{b}$ : coupes axiales en filtre dur montrant les foyers d'ostéolyses à l'emportepièce du squelette axial (flèches jaunes)

L'analyse anatomopathologique des fragments de la tumeur, obtenus après biopsie scanno guidée per cutanée, montrait une prolifération tumorale maligne monomorphe constituée de cellules plasmocytö̈des. Au regard des résultats histologiques, radiologiques et biologiques, le diagnostic de myélome multiple avait été retenu selon les critères de l'IMWG de 2014.

\section{Discussion}

Le myélome multiple est un cancer hématologique rare. Il occupe au Congo la 3ème place après les leucémies et les lymphomes parmi les 
hémopathies malignes répertoriés (Malanda et al., 2013). L'âge moyen au moment diagnostic est de 57 ans (Ngolet et al., 2016). De rare cas ont été décrit dans la littérature avant l'âge de 40 ans. Ce fait clinique, rapporté chez un adulte jeune de 32 ans est le premier cas documenté au CHU de Brazzaville.

Au Congo, $97 \%$ des myélomes multiples sont diagnostiqués au stade symptomatique ou de complication (Ngolet et al., 2016). La cause serait partiellement imputée à la méconnaissance de la pathologie par le personnel médical, au niveau socio-économique bas des patients ainsi qu'au plateau technique diagnostique (Nnonyelum et al., 2015 ; Kariyawasan et al., 2007 ; Ndiaye et al., 2010).

Actuellement, le diagnostic de myélome multiple symptomatique repose sur les critères révisés de l'IMWG de 2014 (Rajkumar et al., 2014) (tableau I).

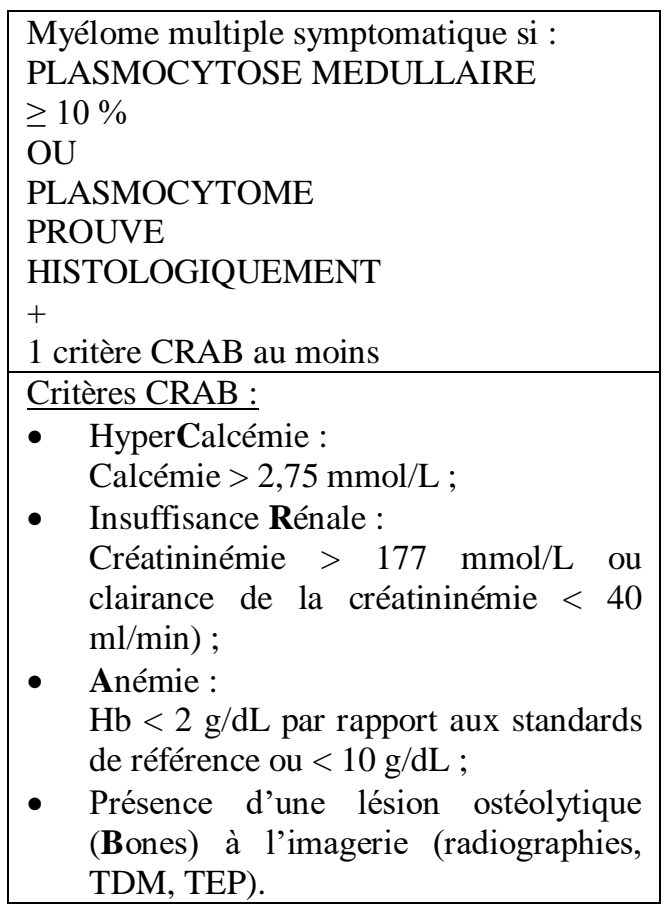

La présence de 3 critères CRAB (hypercalcémie, insuffisance rénale et lésions lytiques multiples du squelette axial) et d'un plasmocytome sacré prouvé histologiquement a permis dans ce contexte de retenir le diagnostic de myélome sans avoir recours aux autres examens biologiques.

Il est décrit qu'en moyenne, $80 \%$ des patients myélomateux, présentent ou vont présenter une atteinte osseuse (Monzani et Felter, 2010). C'est là toute l'importance de l'imagerie médicale. Elle joue un rôle majeur dans le diagnostic des formes précoces en montrant ces atteintes osseuses. Elle permet 
de déterminer le stade évolutif de la maladie et son pronostic. Elle guide la décision thérapeutique et sert également de point initial de référence afin d'évaluer la réponse thérapeutique (Monzani et Felter, 2010).

Le développement de nouvelles techniques d'imagerie plus sensibles et plus spécifiques que les radiographies standards telles que l'IRM corps entier et le Pet-scan a permis d'affiner le diagnostic du myélome multiple. Ces examens rentrent déjà dans la pratique courante de certains centres en occident mais leur accessibilité limitée en Afrique sub saharienne rend difficile d'en recourir en pratique courante.

$\mathrm{Au}$ Congo en particulier, la radiographie conventionnelle, peu couteuse et plus accessible, est le premier maillon de l'arsenal diagnostic radiologique. Elle permet de voir les lésions classiques d'atteinte myélomateuse à type d'ostéolyse à « l'emporte-pièce » des os plats et du rachis sans réaction scléreuse de l'os adjacent. Cependant, il est important de rappeler que ces lésions lytiques du squelette ne sont visibles à la radiographie qu'après une lyse de plus de $30 \%$ de l'os (Monzani et Felter, 2010). La sensibilité des radiographies est faible et cela explique qu'environ $20 \%$ des lésions ne sont pas diagnostiquées avec le risque de sous-estimation de l'étendue de la maladie. Dans cette observation aucune autre lésion du squelette axial n'était visible à la radiographie standard. Les lyses typiques à l'emporte-pièce disséminées au squelette axial attribuable à la maladie plasmocytaire étaient uniquement vues au scanner.

Le scanner est considéré comme l'imagerie alternative de référence pour l'identification des atteintes osseuses (Terpos et al., 2015). Il permet de voir les atteintes infra radiologiques surtout en mode faible dose (low-dose). La mise en évidence de lésions ostéolytiques $\geq 5 \mathrm{~mm}$ au scanner est un critère suffisant pour retenir une atteinte osseuse chez un patient myélomateux.

L'IRM pelvi-rachidienne et l'IRM corps entier (CE) font partie de l'imagerie usuelle lors du diagnostic (Monzani et Felter, 2010). Elles sont plus sensibles et plus spécifiques que les radiographies standards pour la détection des atteintes rachidiennes et pelviennes. L'IRM CE a l'avantage d'explorer l'ensemble du squelette en un seul examen et de remplacer les radiographies standards du squelette entier dont le plus grand inconvénient réside dans l'irradiation qu'elles entrainent. Quand elle n'est pas disponible, il est préférable d'associer aux radiographies standards, une IRM pelvi-rachidienne.

Sur le plan biologique, l'insuffisance rénale et l'hypercalcémie sont des signes de mauvais pronostic de la maladie. L'absence d'anémie clinique et biologique pourrait peut-être expliquer la raison pour laquelle l'état général de ce patient était encore conservé au moment du diagnostic. L'insuffisance rénale est le résultat de la tubulopathie rénale acquise en rapport avec la sécrétion de chaines légères et le dépôt celles-ci au niveau des tubules rénaux. Les troubles hématologiques quand ils sont présents, sont le reflet d'une 
insuffisance de production des lignées sanguines en rapport avec l'envahissement plasmocytaire massif de la moelle osseuse. L'hypercalcémie est en rapport avec les anomalies du métabolisme phosphocalcique, reflet de l'ostéolyse induite par la tumeur.

La localisation du plasmocytome au niveau du sacrum est la seconde particularité de cette observation. Il pose un problème de diagnostic différentiel avec un chordome. C'est la tumeur primitive la plus fréquemment retrouvée dans cette localisation. Les métastases osseuses, les tumeurs à cellules géantes, les lymphomes et les myélomes comme dans notre contexte, sont les autres néoplasies les plus fréquentes du sacrum (Leone A et al., 2010). Aucun critère d'imagerie ne permet formellement de les différencier. Seule l'histologie et le contexte clinico-biologique permettent de poser un diagnostic de certitude.

\section{Conclusion}

Le diagnostic du myélome multiple est plus aisé dans sa forme classique chez le sujet âgé. Les radiographies conventionnelles sont peu sensibles par rapport aux nouveaux moyens d'imagerie diagnostique tels que le scanner et l'IRM. Le scanner, plus accessible en Afrique sub-saharienne, devrait être systématiquement réalisé chez un patient qui se plaint de douleurs osseuses avec des radiographies standards normales. Le recours aux autres techniques d'imagerie doit être méthodique tenant compte de leurs avantages et de leurs inconvénients mais aussi des considérations économiques de nos patients dont les ressources financières sont souvent précaires.

\section{References :}

1. Kariyawasan, C.C. Hughes, D.A. Jayatillake, M.M. Mehta, A.B. (2007). Multiple myeloma: cause and consequences of delay in diagnosis. QJM Mon J assoc Physicians, 100 (10): 635-40.

2. Leone, A. Costantini, A. Guglielmi, G. Settecasi, C. Priolo, F. (2000). Primary bone tumors and pseudotumors of the lumbosacral spine. Rays, Jan-Mar;25(1):89-103.

3. Malanda, J.N. Mbon, J.B.N. Bambara, A.T. Ibara, G. Minga, B. Epala, B.N. Mbalawa, C.G. (2013). Douze années de fonctionnement du registre des cancers de Brazzaville. Bull Cancer, 100(2): 135-9.

4. Monzani, Q. et Felter, A. (2010). Quelle imagerie au cours du myélome multiple ? La Lettre du Rhumatologue, 360: 3-5.

5. Ndiaye, F.S.D. Pouye, A. Fall, S. Diallo, S. Ndongo, S. El Kacimi, S. Diop, T. (2010). Présentation clinique du myélome multiple à Dakar (Sénégal): à propos de 71 observations. J Afr Cancer, 3(1): 8-11.

6. Ngolet, L.O. Kocko, I. Galiba Atipo, F.O. Guelongo Okouango Ova, J.D. Ntsiba, H. Elira-Dokekias, A. (2016). Le myélome multiple 
symptomatique à Brazzaville : à propos de 40 cas. Ann Univ M Ngouabi, 16(1): 1-7.

7. Nnonyelum, O.N. Anazoeze, M.J. Eunice, N.O. Emmanuel, O.O. Stella, A.T. Marcus, A.I. Taiwo, B.M. Olufela, K.O. Chinawaeze, A.J. Orkuma, J.A. Dalhat, G.G. Otobo, U.I. (2015). Multiple myeloma in Nigeria: a multicentre epidemiological and biomedical study. Pan Afr Med J, Nov 24; 22: 292.

8. Rajkumar, S.V. Dimopoulos, M.A. Palumbo, A. Blade, J. Merlini, G. Mateos, M.V. Kumar, S. Hillengass, J. Kastritis, E. Richardson, P. Landgren, O. Paiva, B. Dispenzieri, A. Weiss, B. LeLeu, X. Zweegman, S. Lonial, S. Rosinol, L. Zamagni, E. Jagannath, S. Sezer, O. Kristinsson, SY. Caers, J. Usmani, S.Z. Lahuerta, J.J. Johnsen, H.E. Beksac, M. Cavo, M. Goldschmidt, H. Terpos, E. Kyle, R.A. Anderson, K.C. Durie, B.G. Miguel, J.F. (2014). International Myeloma Working Group updated criteria for the diagnosis of multiple myeloma. Lancet Oncol, Nov; 15(12): e538- 48.

9. Terpos, E. Kleber, M. Engelhardt, M. Zweegman, S. Gay, F. Kastritis, E. van de Donk, N.W. Bruno, B. Sezer, O. Broijl, A. Bringhen, S. Beksac, M. Larocca, A. Hajek, R. Musto, P. Johnsen, H.E. Morabito, F. Ludwig, H. Cavo, M. Einsele, H. Sonneveld, P. Dimopoulos, M.A. Palumbo, A. (2015). European Myeloma Network Guidelines for the Management of Multiple Myeloma-related Complications. Haematologica, Oct; 100(10):1254-66. 